A variance analysis has shown a significant decrease in estradiol levels, while testosterone, LH, SHBG, and FTI levels did not experience any significant change.

According to these findings it can be concluded that the chronic ovarian suppression with buserelin did not influence negatively the androgenic profile.

Laparoscopic Explorative Cyto-Puncture and Aspiration

of Intra-Ovarian Endometriotic Cysts and Deposits 186

Cases in 22 Years

Michel Mintz

Paris, France

$84 \%$ of the patients are between 20 and 40 years old, $70 \%$ are nulliparous or sterile. Among 214 punctured cysts, $62 \%$ contained between 10 and $400 \mathrm{ml}$ (27-91 mm diameter). Judged by 79 surgical controls, the reliability of cytology is $84 \%$ : it eliminated corpus luteum cysts, revealed among 214 ovarian endometriosis almost $50 \%$ of intra-ovarian hidden cases, and contributed to the discovery of 3 endometriotic malign cysts of benign aspect ( 1 false-negative).

Among 186 patients, 24 had complementary surgical cyst resections. 139 patients bearing 155 cysts were followed-up after puncture: 80\% for more than 2 years, $50 \%$ between 6 and 20 years. Most of the prescriptions (33 different gynecologists) were not correctly followed.

Among 109 second-looks and/or ultrasonic controls: 47 cases of persistance or recurrence, 62 showed no cysts, although 15 had revealed cysts from 50 to $100 \mathrm{ml}$. Among 65 patients who wanted children, 31 bore 40 known living children, although 16 had had large punctured cysts of $50-400 \mathrm{ml}$.

\title{
Conclusions
}

This technique seems appropriate to young, nulliparous or sterile women, and should be used before long prescriptions and before surgical resections which do not always prevent recurrence and may cause adhesions.

For elder patients, surgical resection of endometriotic cysts should be performed, since it concerns a risk group of ovarian cancer.

Hormonal Changes during Danol Treatment of Patients

Attila Török, Jôzsef Bôdis, Imre Csaba

Department of Obstetrics and Gynecology, University Medical School of Pécs, Pécs, Hungary 
Fifteen patients were treated with $400 \mathrm{mg} /$ day and another 15 patients with 300 $\mathrm{mg} /$ day of danol. Blood samples were taken before the treatment and in monthly intervals thereafter. Serum luteinizing hormone ( $\mathrm{LH})$, follicle-stimulating hormone (FSH), prolactin, hydroxycortisone, estradiol and progesterone levels were determined with radioimmunoassays.

\section{Posters 160}

The $\mathrm{LH}$ and FSH levels showed a decreasing tendency during the treatment. There was no significant change in the prolactin and hydroxycortisone values. The estradiol and progesterone significantly fell, although when Danol was withdrawn or the dose reduced there was a striking elevation in the estradiol levels.

Based on these results, we suggest that danol, although it was previously thought to have antigonadotroph effects, is acting rather on the steroid receptors or as an inhibitor of the steroid synthesis. At the end of the 6-month danol treatment luteinizing hormonereleasing hormone was given intravenously and caused a significant increase in LH levels which seems to support this hypothesis.

Amenorrhea as a Prognostic Indicator in Endometriosis

Patients Treated with Danazol

J.M. Wheeler, G.O. Patton, M.L. Crowder, M.L. Maccato, L.R. Malinak Houston, Tex., USA

Initial studies advocating the use of danazol for the treatment of infertile women with endometriosis advised achieving a state of amenorrhea. Despite anecdotal evidence that amenorrhea is a good prognostic sign while on danazol, there are few studies examining the issue. The purpose of this study was to determine if failure to obtain amenorrhea was a significant risk factor for persistent infertility in women with endometriosis. All endometriosis patients were operatively diagnosed; 125 were prescribed danazol in dosages individualized due to a $10 \%$ incidence of bothersome side effects. Our therapeutic goal was to achieve 3 months of amenorrhea on no more than $800 \mathrm{mg}$ of danazol daily. Analysis was by frequency analysis using the $\% 2$ distribution. When type of operation for operative diagnosis was compared, the only difference was the propensity of women treated at laparotomy to have more severe endometriosis than those women treated at laparoscopy ( $p-0.001)$. There were no demographic differences between the laparotomy and laparoscopy groups. Of the 67 patients ultimately taking $800 \mathrm{mg}$ of danazol daily, 33 $(49 \%)$ continued to have breakthrough bleeding or spotting. Seven of 58 patients (12\%) who could not advance beyond 400-600 mg daily due to side effects had breakthrough bleeding. Pregnancy rates after 18 months of follow-up were calculated. Pregnancy rates, when analyzed by operation performed and severity of endometriosis, did not significantly differ between the bleeding and non-bleeding patients. 
The Role of Unilateral Salpingo-Oophorectomy in the Conservative Surgical Treatment of Endometriosis

\author{
J.M. Wheeler, M.L. Maccato, L.R. Malinak \\ Houston, Tex., USA
}

Unilateral salpingo-oophorectomy (USO) has been advocated in the woman with severe unilateral adnexal adhesions and endometriosis, yet little data exists as to the effect of USO on postoperative pregnancy rates. Of 514 patients treated with conservative sur 